\title{
Một sơ đồ mới nhằm tăng độ sẵn sàng trong các hệ cơ sở dữ liệu phân tán
}

\author{
Nguyễn Nam Hái \\ khoa Tin học, Trương đại học bách khoa Hà nội .
}

\section{Giới thiệu}

Dối với cơ sờ dữ liệu, khả năng sàn sàng đáp ứng nhu cầu cria người dùng khi khai thác là một vấn đề quan trọng. Trong các hệ CSDL phân táll vấn dề này bị chi phối nhiều hơn bởi nhiêu yếu tố hơn CDSL tập trung. Do vậy, làm thế nào để tăng độ sãn sàng cho các hệ CSDL phân tán là một vấn đề quan tâm nhiều $[1-4,7,8]$. Đã có nhiều giải pháp để tăng độ sãn sàng cho CSDL phân tán. Một trong những giải pháp đó là tồ chức lặp dữ liệu tại một số trạm làm việc. Giá phài trả cho giải pháp này là phải duy trì tính tương thích của các bản sao CSDL khi sảy ra các sự kiện cập nhật CSDL hoặc sự cố tại các trạm làm việc hoặc kênh truyền nối các trạm làm việc.

Đà có một số giao thức (Protocol) điều khiền lặp được đề xuất và những giao thức này khảc nhau ở mức độ và kiểu sự cố mà chúng có thề chịu được. Trong các hề phân tán có hai loại sự cố: hỏng trạm làm việc và hỏng kênh truyển giữa các trạm làm việc. Những sự cố này có thề dẫn đến sự phân hoặch của mạng thành một vài nhóm trong đó các trạm trong cùng một nhóm có thể liên lạc được với nhau nhưng không thể liên lạc được với các trạm làm việc thuộc nhóm khác. Các giao thức duy trì độ sẵn sàng và tímh tương thích của dữ liệu lặp khi mạng bị phân hoạch có thể chia làm hai loại [10]:

+ Loại "lạc quan" (optimistic)

+ Loại "bi quan" (pessimistic) 
Dối với loại bi quan, duy trì độ sằn sàng nhưng không được hy sinh tỉnh tương thích của CSDL [1]. Trong các sơ đồ thuộc loại này việc cập nhật chỉ được thực hiện trên một nhóm trong số các nhóm phân hoạch. Ngược lại, loại lạc quan duy trì độ sằn sàng ngay cá khi phải hy sinh tính tương thích của dữ liệu lặp [3]. Việc cập nhật cơ sở dữ liệu có thể được thực hiện ở một nhóm và do đó sự không tương thích có thể sảy ra nhưng chúng được tim ra và giài quyết khi hợp nhất các nhóm. Xét dưới một góc độ nào đó thì các sơ đồ lạc quan có vè duy trì độ sãn sàng của dữ liệu ở mức cao hơn các sơ đồ bi quan, nhưng có những trường hợp không thề hủy bỏ kết quà của những cập nhật khi đã hoàn thành. Và đối với những trường hợp như vậy sơ đồ bi quan thích hợp hơn. Một giải pháp hay được dùng trong các sơ đô bi quan là "phiếu bâu" (voting) và có thể phân chia làm haj loại:

- Phiếu bầu động.

- Phiếu bầu tînh.

Trong các sơ đồ phiếu bầu, giữa các nhón phân hoạch, người ta chọn lấy một nhóm được phép cập nhật và nhóm này được gọi là nhóm "đa số". Với sơ đồ phiếu bầu tînh nhóm có số phiếu bầu lớn nhẩt sẽ được chọn là nhóm đa số. Với loại phiếu bầu động, quan niệm về nhóm đa số thay đồi cơ bản so với loại phiếu bầu tînh. Trong trường hợp này, khi chọn nhóm đa số ứng với mối phân hoạch thì nhóm được coi là thiêu số trước đó sẽ không được xét đến. Đối với sơ đồ phiếu bẩu động một giao tắc (transaction) có thê cập nhật CSDL tại một trạm làm việc nào đó nếu nhóm chứa hiện thời trạm làm việc đó được coi là nhóm đa số. (Không nhất thiết phài là nhóm có số phiếu bầu cao nhất). Các sơ đố phiếu bẩu động mắc một nhược điền chung là sau một số lần phân hoạch liên tiểp có thể dẫn đến trường hợp không thể cập nhật CSDL tại bất kỳ nhóm nào. Hạn chê đã được khắc phục rất nhiều trong sơ đồ đã được đế xuát bở Jian Tang và N. Natarajan [1] nhờ vào việc lưu lại vết của những phân hoạch liên tiếp trong mạng. Tuy nhiên sơ đổ của Jian Tang và $N$. Natarajan cũng như các sơ đồ phiếu bầu động khác mắc vào tỉnh trạng không giải quyết được là khi nhóm đa số bị phân hoạch thành hai nhóm có số phiếu bầu bằng nhau. Trong trường hợp như vậy khả năng cập nhật CSDL sẽ bị mất. Ta xét ví dụ sau:

Vi dụ ta xét mạng gổ 5 trạm làm việc, tại mối trạm làm việc ta gắn cho một phiếu bấu và giài sư các phân hoạch lần lượt sáy ra như sau:

(1) $\{1,2,3,4\},,\{5\}$.

(2) $\{1,2\}\{3,4\}\{5\}$.

Theo sơ đồ dược đề xuất bởi Jian Tang và N. Natarajà thì sau lần phân hoạch thứ hai các trạm làm việc sẽ có bức tranh về phân hoạch mạng như sau:

Trạm $5:\{1,2,3,4\},\{5\}$, các trạin $1,2,3,4:\{1,2\}\{3,4\}\{5\}$ 
và với những thông tin được biết về mạng như vậy không chọn được nhóm đa số nên không có trạm nào được phép duy trì cập nhật. Do đó sau lần phân hoạch thứ hai thì độ sẵn sàng của CSDL bị naất, việc cập nhật không thể sảy ra tại bất kỳ nhóm nào.

Trong bài báo này chúng tôi đề xuất một sơ đồ mới trên cơ sở phát triển một số ý tường đã được đề xuất trong [1] và [6]. Sơ đồ mới cho phép khắc phục nhược điềm trên của các sơ đồ phiếu bầu động nhờ vào việc xây dựng một quan hệ.tlứ̛ tự trên tập các nhóm các trạm làm việc.

Trong phân hai sẽ trình bầy một số khái niệm cơ sờ cân phục vụ cho việc xây dựng sơ đồ và những chứng minh cần thiết. Phần ba sẽ trình bầy sơ đồ đề xuất. Phần 4 sẽ trình bầy một số chứng minh tính đúng dắn của sơ đồ.

\section{Một số khái niệm cơ sớ}

CSDL phân tán là một tập có cấu trúc các phẩ tư dữ liệu được phân bố trên mạng máy tính. Mồi máy tînh trên mạng gọi là một trạm làm việc, các máy có thể liên lạc với nhau bởi kênh liên lạc hai chiều. Các phẩn tư có thể lặp tại một số trạm làm việc. Trong trường hợp như vậy ta gọi là CSDL hân tán lặp. Trường hợp tại mỗi trạm đêu có đầy đủ các bản sao của các phần tử tạo nên CSDL gọi là CSDL phân tán lặp toàn phẩn. CSDL phân tán lặp toàn phân chính là đối tượng nghiên cứu trong bài báu này.

Đối với CSDL phân tán, tại mỗi thời điêrn có thề có nhiều người khai thác CSDL tại các trạm làm việc khác nhau. Mơi truy nhập tới CSDL có thể xem như bộ ba $\langle R, W, f\rangle$, trong đó $R$ là tập các phẩn tứ dữ liệu đọc, $W$ là tập các phân tư dữ liệu viết, $f$ là hàm mô phỏng những tính toán trong yêu câuu truy nhập. Đề đồng bộ các truy nhập với mục đích duy trì sự tương thích của CSDL có thề sư dụng động hổ logic. Đồng hồ logic là cách thực gán một số nào đó cho một sự kiện sảy ra trong hệ thống đề đánh dấu thời gian sảy ra sự kiện đó [5]. Các đồng hổ logic trong một hệ thóng phân tán phải thóa mân hai điều kiện sau:

1. Nếu $a$ và $b$ là hai sự kiện sáy ra tại một trạm $S_{i}$ nào đó, $a$ sáy ra trước $b$ thì $C_{i}(a)<C_{i}(b)$.

2. Nếu $a$ là sự kiện gựi thông báo bởi $S_{i}$ và $b$ là sự kiện nhận thông báo đó bởi $S_{j}$ thì $C_{i}(a)<C_{j}(b)$.

$C_{i}$ và $C_{j}$ là các đồng hồ logic tại các trạm $S_{i}$ và̀ $S_{j}$. Khi có một hệ thóng đồng hồ logic trong một hệ thống phân tán thỏa mãn hai điểu kiện trên thì ta luân xây dựng được một thư tự tông thể giừa các sụ kiện xảy ra trong hệ thống. Thứ tự đó được xác định như sau: $a$ là sự kiện sảy ra tại trạm $S_{\boldsymbol{i}}, b$ là sự kiện sáy ra tại trạm $S_{j}$, ta nói rằng $a$ sáy ra trước $b$ khi và chỉ khi $C_{i}(a)<C_{j}(b)$ hoặc $C_{i}(a)=C_{j}(b)$ và $S_{i}<^{\prime} S_{j}$. Trong đó quan hệ thứ tự <' là một thứ tự tông thể tùy chọn giữa các trạm làm việc trong hệ thống đang xét. 


\section{Mô hình dề xuất :}

Trong sơ đồ mởi do chuing tôi để xuất, có sự dụng một số ý tường và kiến thức trong [1] và [6] sau đây:

+ Kiến trúc truyền thông của hộ thống trong [1] được giữ lại trong sơ dồ mới.

+ Ý tường lưu lại vết cưa những lấn phân hoạch mạng liên tiếp cũng được giữ lại trong sơ đồ mới, nhưng tại mồi trạm chỉ lưu lại hỉnh ảnh của nhóm chứa nó,

+ ý tưởng phân tán tỉnh toán đối với các cập nhật trong [6] cũng được lưu ý trong sơ đồ cừ chúng tôi.

CSDL được xét trong bài báo này thuộc dạng phân tán lặp toàn phần và mỗi bàn sao được gán cho một phiếu bầu. "

Sau đây là chi tiết sơ dồ đề xuất.

3.1. Thứ tự xác định trịi các nhóm phân hoạch

Gọi:

$n$ : số lượng các trạm làm việc trên mạng,

$N_{1}=\left\{S_{1}, S_{2}, \ldots S_{n}\right\}:$ tập tất cà các trạm làm việc trên mạng,

$2^{N}:$ tập tất cả các tập con của $N$,

S: là tập được sắp toàn bộ với quan hệ thứ tự <'.

Tại môi trạm $S_{i}$ gắn cho một nhàn $x_{i}, x_{i} \in \Omega$ và $x_{i} \neq x_{j}$ với $j=1, n$ và $j \neq i$. Đặt $\sum=\left\{x_{1}, x_{2}, \ldots, x_{n}\right\}$ là tập tất cà các nhãn được găn trên mạng. Gọi $\sum^{*}$ là tập tất cả các từ sinh trên bảng chữ $\sum, \alpha \in \sum$, ký hiệu len $(\alpha)$ là hàm xác định số các xuất hiện của các phẩn từ của $\sum$ trong $\alpha$ và gọi là độ dài cưa $\alpha$. Ký hiệu $\varepsilon$ là tìr có độ dài bằng 0 .

Xây dựng $f$ như sau, $f: \dot{2}^{N} \rightarrow \sum^{*}$ và thóa mãn:

- $x \in 2^{N}, x \neq \emptyset$ và $|x|=m$, trong đó $|x|$ là lực lượng cùa $x$, thì $f(x)=x_{1}^{\prime} x_{2}^{\prime} \ldots x_{m}^{\prime}$ sao cho $x_{i}^{\prime}<x_{i+1}^{\prime}, i=1, m-1$ và $x_{1}^{\prime}, x_{2}^{\prime}, \ldots, x_{m}^{\prime}$ là các nhãn của các trạm làm việc thiết lập nên $x$.

$-x \in 2^{N}$ và $x=\emptyset$ thì $f(x)=\varepsilon$.

Giả sừ $x, y \in 2^{N}$, ta định nghĩa

$$
\max (f(x), f(y))= \begin{cases}f(y) & \text { nếu } \operatorname{len}(f(x))<\operatorname{len}(f(y)) \\ f(x) & \text { nếu } \operatorname{len}(f(y))<\operatorname{len}(f(x)) \\ f(x) & \text { nếu } x_{1}>y_{1} \text { và } \operatorname{len}(f(x))<\operatorname{len}(f(y)) \\ f(y) & \text { nếu } y_{1}>x_{1} \text { và len }(f(x))<\operatorname{len}(f(y)) \\ \text { Không được xác định } & \text { Trong các trường hợp còn lại. }\end{cases}
$$

trong đó $x_{1}$ và $y_{1}$ là hai tiền tố tương ứngtrong $f(x)$ và $f(y)$.

Ta nói:

$$
\dot{f}(x)<^{\prime} f(y) \Longleftrightarrow \max (f(x), f(y))=\dot{f}(y) .
$$


Với hàm $f$ xáx định như trên ta xây dựng dược một quan hệ thư tự trên $2^{\kappa}$. Với quan hệ thứ tự đó, khi một nhóm đa số bị phân hoạch thành hai nhóm $x_{1}$ và $x_{2}$ có số phiếu bầu bằng nhau thì việc chọn nhóm đa số mới được thực hiện theo giài thuật sau:

$$
\begin{aligned}
& \text { if }\left(f\left(x_{1}\right)<\left(f\left(x_{2}\right)\right) \text { Majority }=x_{1} ;\right. \\
& \text { else Majority }=x_{2} ;
\end{aligned}
$$

ơ đây Majority là biến xác định trên $2^{N}$.

Việc xác định một nhóm có phải là một nhóm da số không sẽ được thực hiện ngay tại thời điểm phân hoạch. Khi hợp nliất, uhóm hợp nhất sè chì là nhóm da số nếu một trong hai nhóm tham gia hợp nhất là nhóm đa số.

\subsection{Cáu trúc diề khiền tại mỡ trạm}

Tại mỗi trạm, việc điều kiliển hệ thống dược chia thành hai khối:

+ khối CSDL,

+ Khối truyến thông.

Khối CSDL đàm bảo tất cà nhừng thao tác với CSDI như cập nhật CSDL, điểu khiên song song, phục hồi dừ liệu ...

Khối truiyền thông đảm bảo tầt cả các chức năng truyên thông trong hệ thống. Khi khối CSDL muốn chuyền một thông báo tới một trạı khác, thông báo đó được chuyền xuống cho khối truyền thông và khối truyển thông thực hiện chức năng vận chuyển thông báo đó tới địa chỉ cần thiết trong mạng, cũng vậy khi có một thông báo từ một trạm khác chuyền đến cho trạm. khối truyền thông nhận, xừ lý những yêu cầu cần thiết sau đó chuyên cho khối ('SDL nếu thông báo đó được chuyền đến cho CSDL. Nếu thông báo gửu đến chi liên quan đến khối truyều thông thì thông báo đó được xừ lỵ ngay tại khối trự̂̀n thông chư không cần phải chuyến đển cho khối CSDL. Ngoài ra khối truyền thông còn thụ̣c hiện chức năng phát hiện sự cố trong hề thống theo cơ chế sau: Mỗi trạm sè định kỳ gưu tín hiệu kiền tra tới các trạm láng giểng, nếu sau mộl thời đoạn xác định mà trạm không nhận được tỉn hiệu kiêm tra tư một trạm láng giềng nào đó thì nó coi như kênh truyên tới trạm láng giểng đó đà có sự cố và thông báo sụ kiện này tới tất cả các trạm trong nhóm chứa nó. Cũng bằng cơ chế dó, mồi trạm có thê phát hiện được sự phục hồi của các kênh truyền hoặc bàn thân các trạm láng giềng và thông báo tới tất cà các trạm khác trong nhóm chứa nó.

\subsection{Các biến hệ thống}

Đề duy trì mối liên hệ giừa các trạm trong cùng một nhóm, tại mối trạm làm việc $S_{i}$ trong mạng lưu giữ các biến sau:

$$
\begin{gathered}
+<x_{i}, L_{i}, V_{i}>\text { trong đó: } \\
-x_{i} \text { là nhàn của } S_{i},
\end{gathered}
$$


- $V_{i}$ là một bộ ba $\langle M, V, C\rangle$, trong đó

$+M$ biểu diễn trạng thái của nhó $m$ nhận các giá trị 0 và 1

0 - nhóm thiêu số (Minority),

1 - nhóm đa số (Majority),

$+V$ số hiệu version cập nhật của các bản sao CSDL trong nhóm,

$+C$ tập các trạm làm việc trong cùng nhóm.

- $L_{i}$ là tập các kênh thiết lập nên các đường liên lạcgiữa các trạm trong nhóm chứa $S_{i}$ có dạng $(e, s)$, trong đó:

$+e$ tên của kênh,

$+s$ trạng thái của kênh nhận hai trạng thái 0,1 . Trong đó 0 có nghĩa là kênh còn tốt, 1 có nghía là kênh đã hóng.

+ Label $=\left\{x_{1}, x_{2}, \ldots x_{n}\right\}$ là tập tất cả các nhãn gán cho các trạm làm việc trong toàn mạng.

$+C_{i}$ đồng hồ logic nhận các giá trị nguyên $0,1, \ldots, \infty$ và được khởi đầu là 0 .

\subsection{Giao thức xứ lý sụ cố trền mạng}

\subsubsection{Các thông báo và hàm}

Dể điểu khiên hệ thống khi có sự cố, hệ thống sư dụng các hàm và thông báo sau::

- Hàm Group_Update(e), e là têlı kênh có sự cố sáy ra. Hàm này được gọi là trạm làm việc phát hiện ra sự cố của kênh nối nó với một trạm láng giềng hoặc nó znó nhận được thông báo về sự cố của một kênh nào đó trong nhởm chứa nó.

- Thông báo failed(e,ts), e là tên kênh có sựicố sáy ra, ts là tem thời gian được gán cho bời trạm phát hiện ra sự cố. Thông báo này được sinh ra khi một trạm nào đó phát hiện ra sự kiện kênh có sự cố.

3.4.2 Giao thức xừ lý sự cố

Khi một trạm $S_{i}$ phát hiện kênh nối của nó với trạm láng giềng bị hóng nó sẽ thực hiện:

- gọi hàm Group_Update(e),

Trong đó $e$ là tên'của kênh bị hỏng. Hàm này sẽ thực hiện chức năng cập nhật tại $L_{i}$ và kiêm tra xem sự hóng kênh có dẩn đến phân hoạch không. Nếu sự kiện phân hoạch sảy ra nó sê xừ lý như sau: Nếu nhóm chứa $S_{i}$ là nhóm đa số thì dựa vào số phiếu bâu có được trong nhóm chứa mới và quan hệ thứ tự $<^{\prime}$ trên $2^{N}$ đã xây dựng ở trên. đề xảc định xem nhóm chứa $S_{i}$ mới có là nhơm đa sớ không, đồng thời cập nhật tại $V_{i}$ : Nếu nhóm chứa $S_{1}$ là nhóm thiêu số thì hàm này chỉ phải thực liiện cập nhật $V_{1}$.

$C_{i}=C_{i}+1$.

Gửi thông báo failed(e,ts) tới tất cá các trạm trong nhóm chứa nỏ, ở đây $E$ là "1 của kênh bị hòng, $t s$ là tem thời gian của $S_{i}, t s=\left(C_{i}, i\right)$.

l.i môt tram $S$, nhâi đướ thông báo nàv sè thưc -hiên: 
$+C_{j}=\max \left(C_{j}, C_{i}+1\right)$,

+ Gọi hàm Group_Update(e).

Các hàm này sẽ thực hiện song song với các giao tác khác như các giao tác độc lập; ,không phụ thuộc vào hiện thời trạm đang cập nhật hay hợp nhất.

3.5 Giao thức xư lý cậ nhât CSI)L

3.5.1 Các cấu trúc dữ liệu điều kliển cập nhật CSSL

a) Các hàng đợi:

L. - Global_queue: cho các cập nhật tư xa,

- Local-queue: cho cảc cập nhật phát sinh tại trạm,

- Exec_queue: cho các cập nhật khi có đi điều kiện đề thực hiện.

b) Các dạng thông báo:

- Request $(R, W, f)$ : được CSER gửi đến khi muón thực hiện việc truy nhập cơ sờ dữ liệu.

- Update $\left(R, W, f,\left(C_{i}, i\right)\right)$ : được sinh ra khi nhận yôu cầu cập nhật của USER.

' - Reply(ts): được sinh ra đề trả lợi cho thông báo Ipdate nhận được từ trạm khác.

- $\operatorname{Exec}\left(i, t_{1}, t_{2}\right)$ : được sinh ra khi vêu cầu cập nhật tại trạm $S_{i}$ ờ thời điêm $t_{1}$ đã dù diểu kiện đề chuyền vào hàng đợi thụ̣c hiện. $t_{2}$ là thời gian sinh ra thông báo Exec.

\subsubsection{Mô hỉnh xừ lý cập nhật}

Khi có yêu cầu truy nlıập CSDL tại một trạm $S_{1}$ nào đó, $S_{1}$ sè tăng $C_{1}$ lên 1 , sau đó kiêm tra $W$ có rồng hay không. Nếu $W$ rồng thì yêu câu được chuyên ngay vào hàng đợi thực hiện, ngược lại $S_{1}$ sè kiềm tra xem uhóm chứa nó có phài là nlớm đa số không. Nếu nhóm chứa nó là nhóm thiề số thỉ yêu cầu cập nhật sê bị loại. ngược lại thông báo Update $\left(R, W, f,\left(C_{i}, i\right)\right)$ sè được đặt vào hàng Local_queue. Sau đó nó sê gừi thông báo $\operatorname{~pdate~}\left(R, W<f,\left(C_{i}, i\right)\right)$ tới tất cà các trạm trong nhóm chứa của nó. Trong khoảng thời gian này nếu sạy ra phân hoạch thi các trạm trong̀ uhóm sè xì̀ lý sau:

+ Nểu trạm thuộc nhóm thiêu số thỉ mọi cập nhật trong các hàng dợilocal_queue cũng như trong global_queue dêu bị loại bò,

+ Nếu trạm thuộc nhơm đa số thị việc kiêm tra chì phải xét trong hàng đợi globa_queue. Những cập nlật xuất phát từ những trạm thuộc nhóm thiều số sẽ bị loại còn những cập nhật xuất phát từ những trạm thuộc nhóm đa số vẫn được giữ lại.

Khi một trạm $S_{j}$ nào đó nhận được thông báo Ipdate() tư một trạm $S_{i}$ trong nhóm, nó sẽ thực hiện:

$+C_{j}=\max \left(C_{j}, t+1\right), \gamma \xi$ I

+ đặt thông báo vào hàng đợi global_queue,

+ gưu thông báo $\operatorname{Reply}\left(\left(C_{j}, j\right)\right)$ cho trạm $S_{i}$. 
Khi trạm sinh cập nhật $S_{i}$ nhận được trà lời Reply $(t, j)$ từ trạm $\dot{S_{j}}$ nào đó trong nhóm nó thực hiện:

. $+C_{i}=\max \left(C_{i}, t+1\right)$,

+ Kiềm tra xem đã nhận đư thông báo Reply cần thiết chưa. Nếu đã nhận đủ $S_{i}$ thực hiện:

- Chuyên Update() vào hàng đợi thực hiện exec_queue,

- Loại Update() khỏi hàng đợi local_queue,

- Gừi thông báo exec $\left(i, t_{1}, t_{2}\right)$ tới tất cả các trạm trong nhóm chứa hiện thời cúa nó. Trong đó $t_{1}$ là thời gian khởi sinh cập nhật, $t_{2}$ là thời gian hiện tại của $S_{i}$.

Một trạm $S_{j}$ nào đó khi nhận được thông báo exec $\left(i, t_{1}, t_{2}\right)$ nó thực hiện các hàuh động sau:

$+C_{j}=\max \left(C_{j}, t_{2}+1\right)$,

+ Chuyển thông báo $\operatorname{Update}\left(R, W, f,\left(i, t_{1}\right)\right)$ vào hàng đợi thực hiện Exec_queue, đồng thời loại nó ra khòi hàng đợi Global_queue.

Một Update $(R, W, f,(t, i))$ trong hàng đợi thực hiện Exec_queue được thực hiện khi không có một Update $\left(R^{\prime}, W^{\prime},\left(t^{\prime}, j\right)\right)$ nào trong hàng đợi thực hiện Exec_queue mà:

$-t^{\prime}<t$

- $R^{\prime} \cap W \neq \emptyset$ or $W^{\prime} \cap W \neq \emptyset$ or $W^{\prime} \cap R \neq \emptyset$ or $W \cap R \neq \emptyset$.

\subsection{Gino thức hợp nhất các nhóm phân hoach}

3.6.1 Các cấu trúc dữ liệu điêu khiện hợp nhất

- Thông báo Var_merge(ts, $L, V)$

- Thông báo Group_merge(ts, $L, 1)$

- Thông báo Repare $(\epsilon, t s)$

- Hàm Link_repare(e), e là tên kênh được phục hồi.

- Hàm Adopt_new_var $(L, V)$.

Các thông bạ́o, hàm được sinh ra hoặc gọi tại nhựng thời điểm thích hợp trong quá trình hợp nhất.

3.6.2 Giao thức hợp nhất

Việc khôi phục kênh hoặc trạm có thể dẫn đến việc hợp nhất một vài nhóm với nhau. Khi một trạm hơng thì bao giờ cũng kéo theo hóng một vài kênh và khi trạm được sứ a chữa có thể kéo theo một vài kênh sửa chữa. Do vậy mọi sự kiện hơng hoặc sưa chữa trạm ta đều có thế quy vê sự kiện hỏng hoặc sưa chữa kênh. Cho nên, ta chì cần quan tâm đến các sự kiện đối với kênh là đủ. Khi sảy ra hợp nhất thi nhóm hợp nhất chì có thề là nhóm đa số khi một trong các nhóm tham giam gia hợp nhất là nhóm đa số. Ngược lại hợp nhất sẽ là nhóm thiêu số. Khi trạm $S_{i}$ phát hiện ra kênh liên lạ.c $(i-j)$ được sứa chữa thì tạj trạm $S_{i}$ sẽ thực hiện:

$+C_{1}=C_{1}+1$,

+ Gọi hàm Link_repare $(i-j)$; 
Hàm Link_repare $(i-j)$ sè thực hiện chức năng cập nhật tại $L_{1}$ dồng thời kiềm tra xem sự kiện sưa chựa kênh $(i-j)$ có dân đếu hợp nhất không. Nếu hợp nhất, hàm trả lại giá trị 1 ngược lại trà lại giá trị 0 . Việc kiền tra được tiến hành bằng cách trạm $S_{i}$ sè kiền tra $S_{j}$ có nằm trong nhóm chứa nó không (kiềm tra xem có nằm trong $V_{i} . C$ không).

+ Gựi thông báo sự kiện này tới các 1 rạm trong nhóm clựa nó trì $S_{j}$ bằng thông báo Repare $(i-j, t s)$.

Nột trạm $S_{k}$ nào dó sau khi nhận dược thông báo nà̀ sề thực hị̣̂̂n:

- $C_{k}=\max \left(C_{k}, C_{1}+1\right)$,

- Gọi hàm Link_Repare $(i-j)$, nếu làm này trả lại giá trị l có nghia là sự kiện hợp nhất xảy ra thi trạm tạm dìng tất cà các yêu cầu cập nhật của người ding CSDL đè chuần bị cho quá trinh hợp nhất.

Trong $S_{j}$ và $S i$ chọn lấy một trạm làm trạm phối hợp còn một trạm bị phối hợp việc chọn dụa vào thứ tụ các nhãu cua $S_{i}$ và $S_{j}$ trong mạng. Trạm nào có thứ tự đi trước sè được chộn làm trạm phối hộp, chẳng hạn $S_{i}, S_{i}$ sc gử thông brio: Var_merge $\left(s_{1}, L_{1}, V_{1}\right)$ cho $S_{j} . S_{j}$ sau khi nhậu durọ̣c thông báo Var_merge() sê thục hị̣̂n:

$+C_{j}=\max \left(C_{j}, t_{i}+1\right)$.

+ Giru thông báo Var_merge(t $\left.s_{j}, L_{j}, l_{j}\right)$ ( ho $S_{i}$. sì san khi nhận dựcthông báo đáp lại sè thực hiện:

$+C_{i}=\max \left(C_{i}, t_{i}+1\right)$.

Sau dó tại mời trạm sò tiến hành hợp nhấ dề dựç $(M, I, C)$ mới nhur sau:

$+V . M=\max \left(V_{i} . M, V_{j} . M\right)$.

$+V \cdot C=\max \left(V_{i} \cdot V_{1} V_{j} \cdot V^{\prime}\right)$

$+V \cdot C=V_{i} \cdot C \cup V_{j} \cdot C$.

Mồi trạm thuộc nhóm mới hợp nhất có $L$ nhur sau:

$+L=l_{i} \cup L_{j} \cup\{(i-j) \cdot 0\}$.

Sau dó so sánlı $V_{i}, V$ và $l_{j} . l$. Nếu:

a) $l_{i} \cdot V=l_{j} \cdot V^{\circ}$

$S_{i}$ trụ̂̀n thông báo Group_merge $\left(t s_{i}, L_{i} . l^{-}-i\right)$ cho các trạm lánh giềng của nó trù̀ $S_{j}$. Trạm $S_{k}$ nào dó sau khi nhận dược thơng báo Group_merge $\left(s_{i}, L_{i}, V_{i}\right)$ sè thục hiện:

+ Gọi hàm adop_new_var $\left(L_{i}, Y_{i}\right)$. Hàm nlỳy sẽ thục hiện g’ạ $L_{k}=L_{1}$ và $V_{k}=l_{1}$.

$+C_{k}=\max \left(C_{k}, t_{1}\right)$.

Trạm $S_{j}$ cùng thục hiện turơng tụ.

b) $V_{i} . V \neq V_{j} . V$. Gia sul $V_{i} \cdot V>V_{j} \cdot V$ thực hiện uhu sau:

$S_{i}$ gưi $L_{i}$ và $V_{i}$ tới các trạm trong nhóm cù cua nó. Đối với $S_{j}$ thì ngoài việc gửi $L_{i}, V_{i}$ nó còn phài lan truyền cà CSIDL. Sau dó $S_{j}$ lại thực hiện vưi các trạm trong nhóm cù cua nó như $S_{i}$ đà thực hiện với nó. Tại một thời điểm rhi ró nhiếu nhất 
một tiến trinh hợp nhất được thực hiện. Nếu có sự hợp nhất khác sảy ra thì sự kiện đó chưa dược thực hiện. trong quá trỉnh hợp nhất nếu sảy ra sự kiện phân hoạch hoặc hỏng kênh, trạm thì sự kiện đó vẫn được xừ lý đồng thời. Tại nhìnng trạm sau khi đà hoàn thành hợp uhất, người dùng có thề tiến hành cập nhật CSDL nếu nhóm hợp nhất là nhóm đa số.

\section{Chứng minh tính đưng đắn}

Dề chứng minh được tính đúng đắn của sơ đồ ta phải chứng minh được:

+ Tính nhất quán cua CSDL luân được duy tri tại mối bản sao CSDL.

+ Sơ đồ phải đảm bảo được tỉnh tương thich lặp cuia các bản sao CSDL.

Dề giải quyết được hai vấn đề trên ta sẽ chứng minh một số định lý sau:

Dịnh lý 1. Mọi trạm làm vị̂c trong cừng một nhóm có cùng số hiệu version.

Chứng miinh. Theo cách xây dựng một thuật toán điều khiên việc truy nhập CSDL nói trên, khi có yêu cầu truy nhập CSDL tại một trạm nào đó trong nhóm đa số, trạm đó sè kiểm tra xem yêu cầu truy nhập đó có cập nhật CSDL không. Nếu có, nó sè vận chuyển yêu cầu đó tới mọi trạm trong nhóm. Khi cập nhật có đủ điều khiện đề xếp vào hàng dợi thực hiện tại trạn phát sinh nó thi cập nhật cũng được xếp vào hàng đợi thực hiện ờ mọi trạm khác trong nhóm chứa trạm phát "sinh cập nhật. Hơn mừa thuật toán không sinh ra deadlock. Vấn đề này được giải quyết như sau:

Giả sư $U_{1}, U_{2}, \ldots U_{n}$ là dày cập nhật trong liàng đợi thực hiện. Xây dựng đồ thị có hướng $G=\langle V, E\rangle$, trong dó:

$+V$ là tập đỉnh bao gồm các cập nhật,

+E là tập cạnh được xây dụng như sau:

$$
E=\left\{\left(U_{i}, U_{j}\right), \mid U_{i}<U_{j} \text { và }\left(W_{i} \cap R_{j} \neq \emptyset \text { hoặc } W_{i} \cap W_{j} \neq \emptyset \text { hoặc } R_{i} \cap W_{j} \neq \emptyset\right)\right\} .
$$

Dê dàng chỉ ra được rằng $G$ không ço chu trỉnh. Do vậy thuật toán không sinh ra deadlock, do đó mọi cập nlật khi clã được xếp vào hàng dợi tực hiện đểu được thực hiện. Như vậy version của mọi trạm trong cùng một nhóm là giống nhau.

Dịnh lý. 2. Nêu hai trạm bất kìy trong mạng có số hiệu ve rsion giớng nhauthì hai bản sao CSDL giống nhau hoàn toàn.

Chứng minh. Việc chứng minh địnlı lỷ không phức tạp lắm, nên phẩn chứng minh định lý này không trỉnh bày ờ đây.

Định lý 3. Già sủ $G_{1}, G_{2}$ là hai nhóm nào đó trên mạng. Tại thởi điểm t bất kì̀ nếu $G_{2}$ có version lớn hơn cư $G_{1}$ thi luân luân tồn tại một dãy cộp nhật tác động trên $G_{2}$ và không tác động trên $G_{1}$, có thè chuyĉ̀n $G_{1}$ thành $G_{2}$ nếu nó tác động trên $G_{1}$. 
Chứng minh. Trước hết ta chứng minh định lý với điều kiện không có sự kiện sứa chữa kênh hoặc trạm làm việc, có nghia là không có sự kiện hợp nhất nhóm mà chỉ có sự kiện phân chia nhóm.

Ký hiệu $\operatorname{Ver}(G)$ là version cùa nhóm $G$.

Giả sư $\operatorname{Ver}\left(G_{1}\right)=n$ và $\operatorname{Ver}(G-2)=m(m>n)$, và tại thời điêm $t^{\prime}$ nào đó mà $\operatorname{ver}\left(G_{2}\right)=n$. Vì tại thời điềm $t^{\prime}, G_{2}$ vẫn có thể cập nhật được nên $G_{2}$ là nóm đ̛̣a số và $G_{1}$ là nhóm thiều số. Do giả thiết không có sự kiện hợp nhất sảy ra nên $G_{1}$ không thề trở thành nhóm đa số nên tại thời điêm $t^{\prime}$ thì $\operatorname{Ver}\left(G_{1}\right)=n$. Theo định lý 2 tại thời điềm $t^{\prime}$ các bàn sao CSDL tại $G_{1}$ và $G_{2}$ hoàn toàn giống nhau. Gọi $U_{1}, U_{2}, \ldots, U_{n}$ là dãy cập nhật đã chựền CSDL từ trạng thái có version $n$ đến trạng thái có version $m$, chính là dãy cập nhật cần tỉm đề chuyên $G_{1}$ thành $G_{2}$.

Xét với trường hợp có sự kiện hợp nhất xầy ra. Trong trường hợp có sự lan truyền CSDL thì ta coi như là chịu sự tác động cuia một dãy cập nhật đề chuyên. sang version cao hơn. Dịnh lý được chứng minh.

Như vậy vấn đề tương thich lặp trong sơ đồ đề xuất đã được giải quyết, bây giờ ta chỉ còn phải giải quyết vấn đề nhất quán dữ liệu đối với mối bản sao CSDL nữa là xong. Vấn đề này sẽ được giải quyết bằng định lý 4 dưới đây.

Định lý 4. So đồ đề xuất duy tri tính nhất quán dũ liệu đối với mồi bản sao CSDL. Chứng minh. Phần chứng minh định lý này dề dàng thu được bằng cách chì ra đồ thị "có thể tuân tự hóa được" cuia thuật toán điều khiên cập nhật tại mối trạm làm việc là không có chu trỉnh. Phẩn chứng minh chi tiết không trỉnh bày ở đầv.

\section{Kết luận}

Tóm lại, bài báo trình bày một sơ đồ mới với khả năng chấp nhận được thêm những dạng phân hoạch mới mà các sơ đồ trước đây không chấp nhận. Thêm nữa, thuật toán điều khiến cập nhật CSDL phân tán hoàn toàn được áp dụng và phát triền trong điểu kiện có sự phân hoạch của mạng đã làm tăng độ tin cậy và độ song song trong sơ đồ đã đề xuất.

\section{Tài liệu tham kháo}

1. Jian Tang \& Natarajan N., A scheme for increasing availability in patitioned replicated databases' Informatic Sciences, v. 53. (1991), p. 1-34.

2. Berstein P.A. \& Goodman N., An algorithm for concurrency and recovery in replicated distributed databases, ACM trans. Database systems 9(4):596-6.15 dec. 1984.

3. Davision S.B., Optimism and consistency in patitioned distributed database systems, ACM trans. Database systems 9(3):456-481 sept. 1984. 
4. Parker D.S.,Popek G.J., Rudsin C.. ..., Detection of mutual inconsistency in distributed systems, IEEE trans. sofware eng. SE-9 n. 3 May 1983.

5. Lamport L., Time, clocks and ordering of events in distributed systems' Comm. ACM, July 1978 , p. 558-565.

6. Singhal M., Update transport: a new technique for update synchronization in replicated database systems, IEEE trans. sofware eng., v. 16, n. 12 Dec. 1990.

7. Tamer M., GTE laboratories, Patrick Valduricz, INRIA, Distributed database systems: Where are we now?, IEEE trans. computer, August 1991.

8. Berstein P.A. \& Goodman N., Concurrency control in distributed database systems, Computing serveys, v. 13, n. 2, June 1981.

9. Kumar V., Perfomance comparisionof database concurrency control mechanisms based on two-phase locking, timestamping and Mixed approach, Information sciences, v.51(1990), p. 221-261.

10. Davidson S.B., Molina II.G. \& Skeen D., Concurrency in partitioned networks, ACM comput. serveys 17(3) Sept. 1985.

\section{Abstract}

A new scheme for enhancing availability in distributed database systems

This paper presents a new schcme that enhances the availability of data in the full replicated distributed database systems. This scheme allows to tolerate a new type of the partition of the network that the old previous schemes in the same type doesn't allow. In addition, the update is based on a full distributed algorithm, i.e. every update is performed by all workstations, so paralcllism of computations is higher than schemes using semi-distributed algorithm. This paper also offers a correctness proof of the sch, 